\title{
Mass Media y consumo musical en estudiantes de enseñanza secundaria en Brasil*
}

\author{
JOÃO FORTUNATO SOARES DE QUADROS JÚNIOR** \\ Universidade Federal do Maranhão (Brasil) \\ joaofjr@gmail.com
}

Recepción: 23 de noviembre de 2016.

Aprobación: 20 de febrero de 2017.

Forma de citar este artículo: Quadros Júnior, J. F. S. de (2017). Mass Media y consumo musical en estudiantes de enseñanza secundaria en Brasil. Cuadernos de Lingüística Hispánica, (30), 187-209. doi: https://doi.org/10.19053/0121053X.n30.0.6194

* Artículo de investigación derivado de la tesis doctoral "Preferencias musicales en estudiantes de enseñanza secundaria en Brasil: El caso de la ciudad de Vitória, Espírito Santo". Este fue presentado como conferencia en el "II Coloquio Internacional. Análisis de Discursos Contemporáneos: Cultura y Poder”, promovido por la Universidad Pedagógica y Tecnológica de Colombia (UPTC).

** Doctorado y Maestría en Educación Musical por la Universidad de Granada (España). Maestría en Música por la Universidade Federal da Bahia (Brasil). Coordinador del Grupo de Estudos e Pesquisa "Arte, Cultura e Educação" (GEPACE). Profesor del Departamento de Artes y del Master Profesional en Gestão de Ensino da Educação Básica de la Universidade Federal do Maranhão (Brasil). Producción apoyada por la Fundação de Amparo à Pesquisa e ao Desenvolvimento Científico e Tecnológico do Maranhão (FAPEMA). 


\section{Resumen}

El presente artículo tiene como objetivo principal discutir la influencia de los mass media y de otros agentes de la educación informal en las preferencias musicales de estudiantes de enseñanza secundaria en Brasil. Como datos empíricos, fue desarrollado una investigación con 940 estudiantes (mujeres y hombres) de Vitória (Espírito Santo-Brasil), los cuales confirmaron los mass media como los principales agentes determinantes en su predilección musical, entre los cuales se destacan el internet y la radio, seguidos por la influencia de los amigos y de la televisión. Por lo tanto, se enfatiza la importancia de crear políticas públicas que favorezcan el acceso y difusión musical por diferentes medios, valorizando especialmente su inserción en la educación formal y no-formal, hecho que probablemente suavizaría la potencial soberanía de los agentes mediáticos.

Palabras clave: Música, mass media, adolescentes, educación informal.

\section{Mass Media and Music Consumption in High School Students from Brazil}

\section{Abstract}

The main objective of this article is to discuss the influence of mass media and some other non-formal education agents in the musical preferences of high school students in Brazil. The research was developed with 940 students (men and women) from Vitória (Espirito Santo - Brazil). They confirmed mass media to be the key agent determining their musical preferences, being the internet and the radio the most relevant, followed by T.V and peer influence. This fact emphasizes the importance of creating new public policies that favor the access and dissemination of music through different media, giving a special value to its insertion in formal and non-formal education. The importance of this strategy lies in the fact that it would probably help moderate the potential dominance of media agents over educational figures and institutions.

Key words: music, mass media, teenagers, informal education. 


\section{Médias et consommation musicale chez les élèves dans l'enseignement secondaire au Brésil}

\section{Résumé}

Cet article a comme objectif principal celui de discuter l'influence des médias et d'autres agents de l'éducation informelle dans les préférences musicales des élèves de l'école secondaire au Brésil. Servant comme des données empiriques, on a développé une recherche avec 940 élèves (garçons et filles) de Vitória (Espírito Santo, Brésil), ceux qui ont confirmé les médias comme les principaux agents déterminants dans leur prédilection musicale. On y met en relief l'Internet et la radio, suivis par l'influence des amis et de la télévision. Par conséquent, on souligne l'importance de créer des politiques publiques qui favorisent l'accès et la diffusion musicale par de différents moyens, en valorisant spécialement leur insertion dans l'éducation formelle et non-formelle, un fait qui probablement adoucira la potentielle souveraineté des agents médiatiques.

Mots clés: Musique, médias, adolescents, éducation informelle.

\section{Meios de comunicação e consumo de música em alunos do ensino secundário no Brasil}

\section{Resumo}

0 principal objetivo deste artigo é discutir a influência dos meios de comunicação de massa e outros agentes da educação informal sobre as preferências musicais dos estudantes do ensino médio no Brasil. Como dados empíricos, foi realizada uma pesquisa com 940 alunos (mulheres e homens) de Vitória (Espírito Santo-Brasil), que confirmaram os meios de comunicação de massa como os principais agentes determinantes em sua predileção musical, entre os quais a Internet e a Rádio, seguiram pela influência de amigos e televisão. Portanto, é enfatizada a importância de criar políticas públicas que favoreçam 0 acesso e a disseminação de música através de diferentes meios de comunicação, especialmente enfatizando sua inserção na educação formal e não formal, fato que provavelmente suavizará a potencial soberania dos agentes de mídia.

Palavras-chave: Música, mídia de massa, adolescentes, educação informal. 


\section{Introducción}

La educación informal es concebida como el ámbito o la dimensión de la educación con un carácter no intencional y que comprende influencias, tanto del medio natural como del social, que afectan al desarrollo del individuo (Santos, 2006). Este tipo de educación guarda una innegable relación con los medios de comunicación de masas, los mass media, responsables de gran parte de los mensajes de todo tipo que son recibidos diariamente en los hogares de millones de ciudadanos de todos los países, en las calles de las ciudades y en establecimientos diversos (Lorenzo, 2002).

Avanzar en la comprensión de la influencia personal y social de este tipo de educación, requiere explorar el concepto de mass media, definido por Lorenzo (2002) como el "conjunto de medios de información y/o comunicación que de forma masiva envían mensajes verbo-icónicos a grandes grupos de población" (p. 239) y que ejerce una influencia decisiva en la educación social de los ciudadanos a partir de tres canales informativos: los de mensajes impresos (como los libros, las revistas y los periódicos); los de mensajes fílmicos (películas y fotografías); y los de mensajes electrónicos (televisión, radio, internet, etc.) (Lorenzo, 2004).

Según Merrill, Lee y Friedlander (1991), los mass media poseen cuatro características principales:

a) llegan a un gran número de personas;

b) se da una gran dispersión geográfica de esas personas;

c) existe un anonimato entre los individuos que nutren el grupo del público y la identidad del comunicador;

d) hay gran heterogeneidad del público, entre el que sus miembros presentan identidades muy variadas con relación al sexo, edad, nivel educativo, profesión, afección, inclinación política, etc.

La influencia de los mass media en la sociedad viene siendo constatada desde las primeras décadas del siglo XX por diferentes autores (Slater y Henry, 2013; Vázquez, 1997), algunos de los cuales consideraban a los mass media como un fenómeno perjudicial para el desarrollo de las sociedades, puesto que homogeneizaban las culturas volviéndolas igua- 
les y minimizando sus particularidades. Además, se apuntaba que la cultura, en todos sus niveles, se vería vulgarizada y los medios acabarían influyendo fuertemente y de manera negativa en el comportamiento y los valores humanos. Así, la cultura se transformaría en una gran maraña de clichés altamente previsible (Adorno y Horkheimer, 1985). Sin embargo, otros autores destacan beneficios que solo fueron posibles a partir del surgimiento de estos vehículos comunicacionales. Así, Canclini (2000) afirma que:

Sin la radio, escribieron Carlos Mosiváis y Jesús Martín Barbero, no habría sido posible la formación de naciones capaces de integrar modos de hablar de diferentes regiones, ni habrían surgido líderes populistas que han sabido utilizarla para comunicarse con todas las clases sociales. (p. 46).

En este sentido, analizando los efectos de los medios de comunicación sobre la población, McLuhan (1996) planteó la hipótesis de que el planeta acabaría siendo una 'aldea global' en muchos aspectos de la vida de las personas que lo habitan, de tal manera que buena parte de las actividades productivas y de ocio en que ocupamos nuestro tiempo se verían homogeneizadas por la imposición de patrones de todo tipo: económicos, culturales, sociales, etc. Así, las diferencias culturales entre los pueblos irían siendo eliminadas progresivamente, dando lugar a un proceso natural de aculturación (Laraia, 2002; North y Hargreaves, 1997).

La hipótesis de McLuhan (1996) establece conexión con las ideas de Michel Foucault sobre las relaciones de poder. Foucault (1979; 2008) concibe el poder como una forma de control social sistemático establecido a partir de relaciones de fuerza entre individuos de una sociedad que unas veces detiene y, en otras ocasiones, sufre su acción. El poder no se establece solamente como un mecanismo de fuerza de una macro a una microestructura, sino que está presente en microrelaciones que coexisten en toda sociedad (ej. familia, escuela, iglesia, entre otras). Para el autor, ese proceso es demasiadamente dinámico, ya que todo el tiempo se crean nuevas relaciones de poder en función de las necesidades y realidades de cada local.

Sarramona (1994) propone una síntesis del proceso de transmisión de mensajes provenientes de los mass media y su influencia en los destinatarios de los mensajes, afirmando que esa influencia ocurre de dos maneras:

- incorporándose al acervo cognitivo del destinatario, en caso de que aún no formase parte de él; 
- modificando sus concepciones preexistentes sobre la información recibida, si es que esta ya estaba presente, en alguna medida, en el conjunto de sus conocimientos.

Por su parte, Saperas (1987) apunta que los efectos de la comunicación que llevan a cabo estos medios no solo son de tipo cognitivo, sino que se extienden igualmente al plano de las actitudes y de la conducta, considerando los mass media como elementos principalmente responsables de la construcción de la realidad social (North y Hargreaves, 1997). Por lo tanto, la construcción mediática de la realidad que envuelve actualmente al ciudadano, es tanto ideológica como de comportamiento y, de igual manera, aumenta su poder de influencia en el individuo de forma inversamente proporcional a su capacidad crítica para reducir y controlar la influencia de lo percibido (Costa et al., 2012; Lorenzo, 2002). De este modo, serán sin duda más vulnerables a los efectos de los medios las personas con menor visión analítica del entorno audiovisual, que está directamente relacionada con una insuficiente 0 inadecuada formación académico-cultural (general y específica) para la recepción crítica de los medios, y con las etapas de mayor plasticidad cerebral (infancia y niñez) y modelado de actitudes y conductual (pre-adolescencia y adolescencia) (Cremades, 2008; North y Hargreaves, 1999).

Basado en todo lo expuesto anteriormente, es posible afirmar hoy que la educación informal está totalmente capitalizada por el discurso de los mass media, sobre todo en uno de los principales colectivos consumidores de medios de comunicación: los adolescentes. Este asunto ha sido objetivo de varias investigaciones recientes (Cremades, 2008; Fittipaldi, 2005; Hargreaves y North, 1997; Leung, 2004; Lorenzo y Cremades, 2008; North y Hargreaves, 1999; Quadros Jr. y Lorenzo, 2015; Silva, 2006), muchas de las cuales afirman que los mass media actúan como instrumento de influencia en la formación y consolidación de preferencias musicales en los adolescentes (Fittipaldi, 2005; North y Hargreaves 1997), de comportamiento (Lorenzo 2002, 2003; North y Hargreaves, 1999), e incluso de orientación profesional en este colectivo (Silva, 2006).

El consumo musical está directamente vinculado al concepto de preferencia musical. Schäfer (2008) la define como el grado del gusto por un estilo musical, sumado a la tendencia comportamental para escuchar un estilo más que otros, pudiendo permanecer o desaparecer esta tendencia a lo largo del tiempo. De acuerdo con Levitin (2007), las "preferencias musicales empiezan a ser formadas en el útero materno y son producto de una compleja interacción entre nature y nurture, o sea, entre la personalidad de cada persona y el ambiente en que vive" (p. 4). 
Quadros Jr. y Lorenzo (2010) afirman que la construcción personal de las preferencias musicales puede ocurrir tanto de manera autónoma, a partir de la elección consciente del individuo de lo que quiere escuchar en su cotidianeidad; como de modo inducido, a partir de la escucha involuntaria por imposición del medio o por influencia de otros agentes.

Quadros Jr. y Lorenzo (2016) señalan diversos factores que ejercen influencia en la construcción de la preferencia musical personal, tales como: parámetros específicos de la música (tiempo, ritmo, tono, entre otros); familiaridad, complejidad y audición repetida; experiencias afectivas del oyente mientras escucha música; influencias sociales y culturales; personalidad del oyente; uso de la música; clase social; sexo y edad de los oyentes. Schäfer (2008), por otro lado, destaca también que existen dos abordajes principales que pueden explicar el desarrollo de las preferencias musicales cuando son influenciadas por factores externos al oyente. La primera de ellas es la tendencia: las personas tienden a buscar grupos sociales que comparten opiniones, valores, creencias o hábitos semejantes a los suyos (Finnäs, 1989a). Eso coincide con la idea de adaptación a un grupo (Levine y Russo, 1987); es decir, si nuestras opiniones personales coinciden con las del grupo social al cual pertenecemos, esperamos tener una mayor aceptación (estatus más elevado dentro del grupo); en caso contrario, esperamos algún tipo de exclusión del grupo (o un estatus menos reconocido dentro de este). La segunda es llamada de "influencia informacional" o "efecto prestigio" (North y Hargreaves, 2008): las preferencias por piezas o estilos musicales desconocidos pueden ser influenciadas por la opinión de otros o por la descripción realizada por el propio compositor. North y Hargreaves (2008) afirman que en el caso de que una persona sea informada que Mozart es el autor de una música que desconoce, probablemente su gusto por esa música será mayor que si hubiera sido informado de que la composición pertenecía a un compositor desconocido (Radocy, 1975).

Una vez expuesto, de modo general, el tipo de influencia social que los medios de comunicación ejercen, especialmente en el ámbito de la educación informal, y de qué forma se da la construcción de la preferencia musical (que resultará en el consumo musical), se realiza a continuación un análisis (general, y de modo particular con foco en la realidad brasileña) más concreto de los principales agentes mediáticos considerados en este trabajo: radio, televisión e internet.

\section{Radio}

Creada a finales del siglo XIX, podría decirse que ha sido, hasta fechas recientes, considerado el medio de comunicación más universal (Legorburu, 2001). Según Calabre (2002), la radiodifusión como un servicio de transmisión normalizada surgió en EE.UU. 
en 1920, obteniendo una difusión inmediata en todos los países del mundo. Su popularidad tiene que ver principalmente con la funcionalidad: bajo costo, tamaño reducido, autonomía y movilidad (Silva, 2012).

Legorburu (2001) resume en cinco las características que determinan la identidad de la radio en cuanto medio de comunicación: inmediatez, fugacidad, capacidad de sugestión, incapacidad de elección y limitación de tiempo. Sobre la primera, dice este autor que la noticia se difunde a los espectadores casi de forma inmediata, sin necesidad de mucha infraestructura. La fugacidad se refiere a la necesidad del mensaje de ser oído y comprendido de forma instantánea: "en la radio no existen las segundas oportunidades" (p. 132). La capacidad de sugestión se genera en virtud de la carencia del atractivo visual imaginado, pues exige que el oyente cree mentalmente la imagen de los hechos. La cuarta característica se refiere al hecho de que en la radio (como en la televisión) no se puede elegir el momento exacto para escuchar la noticia o un determinado programa, puesto que existe un orden programado para eso que debe ser seguido. Por último, el horario de la programación debe ser organizado a lo largo de las horas del día, no siendo posible disponer de más tiempo aunque fuera necesario, al revés de lo que ocurre con la prensa escrita, que puede aumentar o disminuir el número de páginas a su conveniencia.

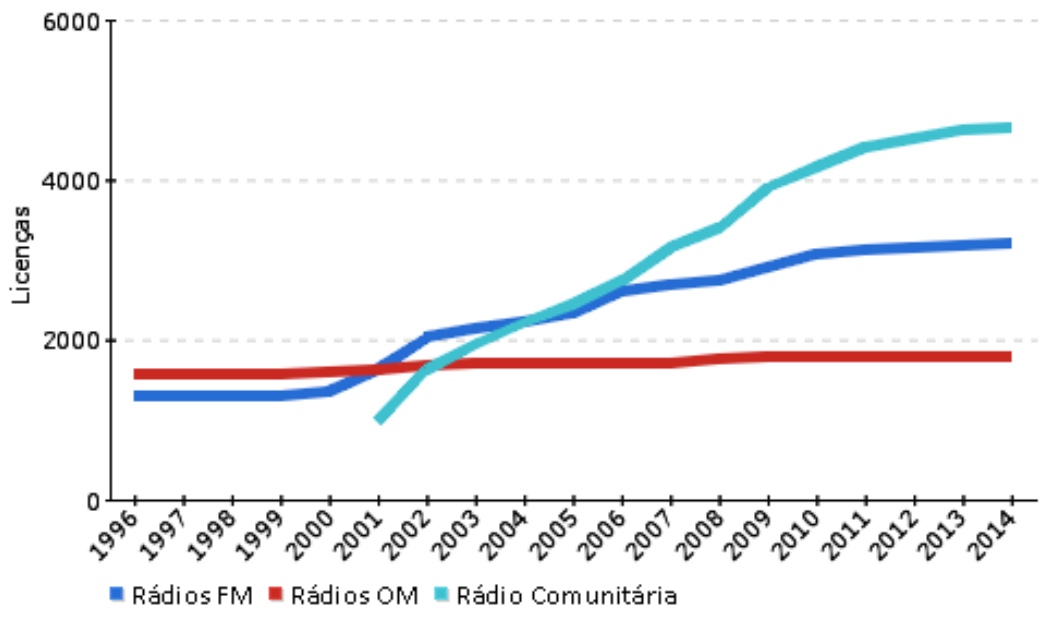

Figura 1. Número de licencias de radio en Brasil: 1996-2014.

Fuente: Telebrasil (2016).

Los datos de una investigación reciente realizada en Brasil, demuestran que en los últimos 20 años hubo una creciente vertiginosa en el cuantitativo de licencias para radios comunitarias (ver figura 1). En sitios lejanos de Brasil o en comunidades situadas en la periferia de las grandes ciudades, donde el acceso al teléfono es difícil, las radios comuni- 
tarias son los medios más rápidos para transmitir cualquier tipo de noticia. Como expone Henriques (2005):

La proliferación de las radios comunitarias y las radios libres y clandestinas, con sus variadas características, es un factor nuevo que precisa ser considerado con relación a la movilización de las comunidades, una vez que este vehículo pasa al primer plano como la principal fuente de información sobre los asuntos locales. (p. 3).

A través de los tiempos, la radio (el aparato físico) ha cambiado de forma, adecuándose a las exigencias de los consumidores y al desarrollo tecnológico, estando accesible en la actualidad por medio de los teléfonos móviles, en los coches o en los ordenadores. Además, pasó también por adaptaciones en los formatos de los programas, tornándose más interactiva, permitiendo la participación efectiva del oyente. Posiblemente, como ya ha sido mencionado por Legorburu (2001), por su capacidad de adaptación, la radio consigue mantener viva su presencia en la sociedad actual.

\section{Televisión}

Considerada como uno de los vehículos e instrumentos sociales más importantes para la transmisión de la información, del entretenimiento y de la formación (Gunter, 2005). Esta, conjuntamente con la radio, constituye el conglomerado llamado Radiodifusión, concebida como un servicio de distribución directa que es libremente recibido por el público (Teleco, 2012).

La evolución tecnológica encabezada por la televisión ha impuesto, según Fernández (2010), dos tipos de dictadura a los contenidos que se difunden por este medio. La primera, la dictadura de la velocidad, que generó en la sociedad el pensamiento de que tiempo es dinero, como afirmaba Benjamin Franklin. La segunda dictadura es la del espectáculo, relacionada con la importancia de la imagen unida a la necesidad de entretener. Para Fernández (2010), "los medios audiovisuales buscan el efecto en los sentidos externos (la vista y el oído), no en el sentido interno, que es la conciencia” (p. 100). Esto es resultado de la urgencia por competir y captar el mayor número de espectadores, enviando mensajes cada vez más rápido al público y no permitiendo reflexiones o preguntas hacia estos.

La televisión se ha configurado como el principal (cuando no el único) medio de acceso a la información para la gran mayoría de la población mundial, lo que le da un sorprendente poder de manipulación en la búsqueda de sus propios intereses. Por ejemplo, en Brasil, está presente en casi todos los hogares (97,2\%), comprobando su gran inmersión en la vida de las personas (ver figura 2). 


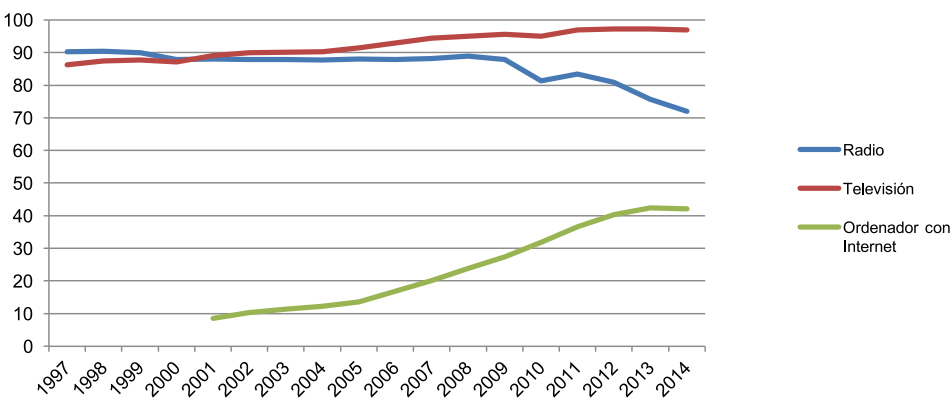

Figura 2. Porcentaje de inmersión de Radio, Televisión y Ordenador con Internet en los hogares brasileños. Fuente: Telebrasil (2016).

Por todo lo anterior, diferentes autores coinciden en la necesidad de que las emisoras de televisión ofrezcan una programación de calidad que ayude en la formación de usuarios críticos y conscientes de los mass media contemporáneos (Carvalho, 1999; Díaz, 2010; García y López, 2010). Un diagnóstico interesante y que muestra el cambio que ocurrió en el consumo televisivo en los últimos 20 años está en el crecimiento del número de usuarios de televisión de pago en Brasil (disponible por cable, radio, satélite o fibra óptica), un servicio que supuestamente ofrece mayor calidad, pluralidad y libertad para el telespectador (ver figura 3).

La popularización de la televisión por suscripción en Brasil está vinculada también al desarrollo económico ocurrido en el país a partir de los años 2000. Así mismo, es posible afirmar que la predominancia actual de la tele como principal medio de comunicación está siendo amenazada por el creciente desarrollo del internet, sobre todo entre los adolescentes. El internet sí tornó la gran ventana mundial de difusión de información y conocimiento desde finales del siglo XX. Internet llegó para cambiar todo el entorno de trabajo hasta entonces conocido por los otros medios de comunicación masivos.

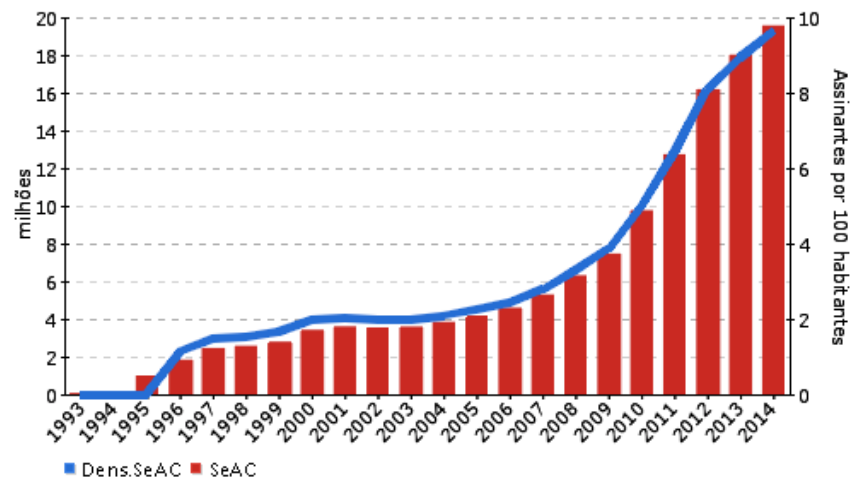

Figura 3. Cantidad (en millones) de usuarios de televisión de pago en Brasil. Fuente: Telebrasil (2016). 


\section{Internet}

Surgido a finales de 1960, la internet puede ser definida como un conjunto descentralizado de redes de comunicación interconectadas que comparten información mediante el uso de la familia de protocolos TCP/IP, garantizando que las redes físicas heterogéneas que la componen funcionen como una red lógica única y de alcance mundial (Liarte, 2000; Monteiro, 2001).

La International Telecommunication Union ha publicado recientemente un estudio sobre el número de usuarios de Internet en el mundo durante la última década (Telecom, 2016).

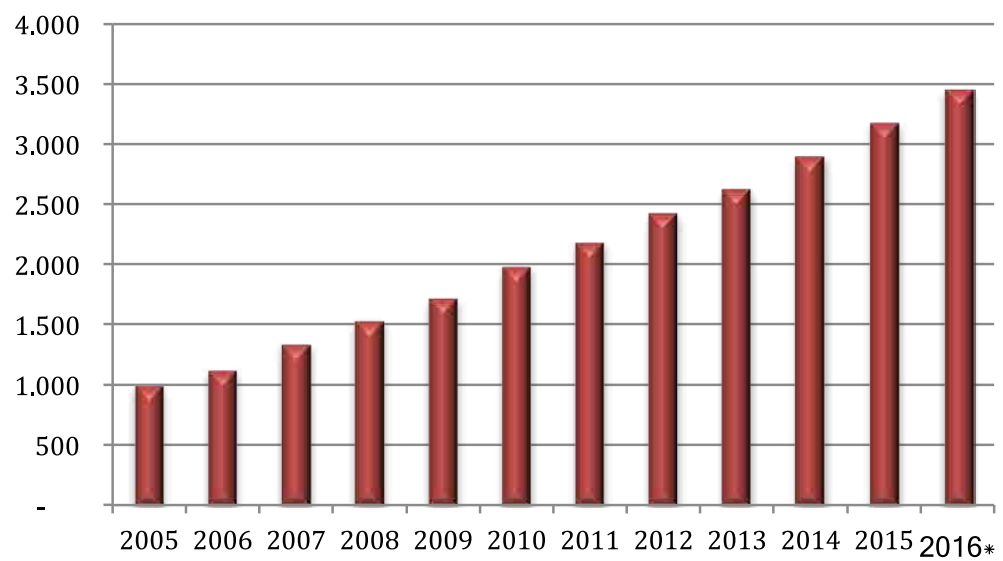

Figura 4. Cantidad (en millones) de usuarios de internet en el mundo. Fuente: ITU World Telecommunication/ICT indicators database.

A partir de la figura 4, se observa que casi la mitad de la población mundial son usuarios de internet, ocurriendo un crecimiento aproximado de $250 \%$ en 10 años. Eso comprueba que la Internet gana cada vez más espacio en el contexto social, ofreciendo un gran abanico de opciones y servicios a la población, desde juegos infantiles hasta compras virtuales. Cremades (2008) apunta diversas actividades cotidianas (de carácter económico, educativo, social y cultural) realizadas actualmente a través de Internet:

- Gestión bancaria;

- Negocios bursátiles a escala mundial;

- Búsqueda de información en bases de datos o mediante potentes buscadores gratuitos; 
- Presentación de solicitudes, registros y diversos documentos mediante aplicaciones telemáticas;

- Servicio de envío de documentos de texto, imágenes, etc. (correo electrónico -e-mail-);

- Compra y venta de diferentes productos a través de la red (comercio electrónico o e-commerce);

- Servicios para toda clase de información y transacciones como la reserva de billetes para transportes, hoteles, vacaciones, cines, actividades artísticas y de ocio en general;

- Realizar la compra de casa en supermercados virtuales;

- Juegos interactivos en red con otros internautas;

- Escuchar, visualizar y descargar música y películas;

- Intercambio de archivos;

- Relacionarse con otras personas y hacer amigos manteniendo conversaciones en tiempo real en diversos foros a través del Chat;

- Pasar consultas médicas en red. (pp. 136-137).

De esta forma, Internet se ha transformado en un mundo paralelo al real, en el que las nuevas redes sociales (Facebook, Twitter, MySpace, etc.) han creado formas diferentes de socialización e interacción.

A partir del recorrido teórico expuesto, se pretende en este análisis discutir la influencia de los mass media (radio, televisión e Internet) y de otros agentes componentes de la educación informal en el consumo musical de estudiantes de enseñanza media en Brasil. A continuación, se presenta el método utilizado para llevar a cabo la investigación.

\section{Método}

\subsection{Participantes}

En esta investigación, participaron 940 estudiantes provenientes de las 13 escuelas estatales de enseñanza secundaria de la ciudad de Vitória (Espírito Santo, Brasil), con edades comprendidas entre los 14 y los 20 años (media de 16.14 años), siendo $56.4 \%$ mujeres y $43.6 \%$ hombres. 


\subsection{Instrumento}

Para la recogida de datos, fue utilizada una versión adaptada al contexto brasileño, específicamente al municipio de Capixaba, del Cuestionario sobre preferencia de estilos musicales (Quadros Jr., 2013). La adaptación se realizó respetando los criterios psicométricos de fiabilidad ( $\alpha=.873$ ) y validez por juicio de expertos (Barbero, Vila y Suárez, 2003).

El instrumento adaptado se articula en torno a la evaluación de la influencia de 8 agentes de educación informal en el consumo musical de los adolescentes (consumo medido a través de las variables escucha, compra y/o grabación de música): Televisión, Radio, Internet, Familia, Amigos, Iglesia, Sitios públicos y Conciertos en vivo.

\subsection{Procedimiento}

El cuestionario fue aplicado a los alumnos con el conocimiento y aprobación de los órganos directivos de los centros educativos participantes. Se administró en sesiones de 50 minutos.

\subsection{Análisis de datos}

Fueron empleados los cálculos estadísticos descriptivos de tendencia central (media) y los test Kolmogorov-Smirnov (para verificación de normalidad de la distribución de los datos), $U$ (de Mann-Whitney) y el Kurskal-Wallis, utilizando como variables de estudio el sexo y la edad.

\section{Resultados}

La estadística descriptiva sobre la influencia de agentes de educación informal en el consumo musical se presenta en la tabla 1. Los resultados generales obtenidos para los agentes evaluados muestran la fuerte influencia de los ítems Internet, radio y amigos, siendo los únicos que presentaron mediana 4. Por otro lado, se observó la poca influencia de la música vehiculada en sitios públicos, como parques, cines, centros comerciales, etc., en las preferencias musicales de los estudiantes, siendo el único ítem que ha obtenido mediana 2 (ver tabla 1). 
Tabla 1. Estadística descriptiva y el test Kolmogorov-Smirnov (K-S) para evaluar la normalidad de la distribución de datos.

\begin{tabular}{llllllllll}
\hline \multicolumn{1}{c}{ Ítem } & Media & Mediana & Min & Max & $\begin{array}{c}\text { Desv. } \\
\text { Típica }\end{array}$ & Variancia & Asimetría & Curtosis & K-S \\
\hline Amigos & 3.38 & 4 & 1 & 5 & 1.283 & 1.645 & -.398 & -.889 & $.000^{*}$ \\
$\begin{array}{l}\text { Conciertos en } \\
\text { vivo }\end{array}$ & 3.06 & 3 & 1 & 5 & 1.455 & 2.118 & -.069 & -1.349 & $.000^{*}$ \\
Familia & 2.83 & 3 & 1 & 5 & 1.433 & 2.053 & .215 & -1.278 & $.000^{*}$ \\
Iglesia & 3.05 & 3 & 1 & 5 & 1.526 & 2.330 & -.052 & -1.449 & $.000^{*}$ \\
Internet & 3.69 & 4 & 1 & 5 & 1.461 & 2.134 & -.733 & -.829 & $.000^{*}$ \\
Sitios públi- & 2.61 & 2 & 1 & 5 & 1.348 & 1.818 & .415 & -.991 & $.000^{*}$ \\
cos & & & & & & & & & \\
Radio & 3.58 & 4 & 1 & 5 & 1.461 & 2.134 & -.666 & -.955 & $.000^{*}$ \\
Televisión & 3.27 & 3 & 1 & 5 & 1.382 & 1.909 & -.332 & -1.126 &, $000 *$ \\
\hline
\end{tabular}

*Estadísticamente significativo al nivel 5\% $(\mathrm{p}<.05)$.

El test de Kolmogorov-Smirnov (K-S) fue empleado para verificar la distribución de los datos (ver tabla 1). Así, se observa que los datos de todos los ítems evaluados no siguen una distribución normal $(\mathrm{p}<.05)$, siendo necesario el empleo de pruebas no paramétricas para realización de los análisis en función de las variables de estudio (sexo y edad).

Tabla 2. Estadísticos descriptivos y test Kolmogorov-Smirnov (K-S) para evaluar la normalidad de la distribución de datos en función de la variable sexo.

\begin{tabular}{|c|c|c|c|c|c|c|c|c|c|c|}
\hline \multirow[b]{2}{*}{ Ítem } & \multicolumn{5}{|c|}{ Femenino } & \multicolumn{5}{|c|}{ Masculino } \\
\hline & $\mathrm{N}$ & Media & Mediana & $\begin{array}{l}\text { Desv. } \\
\text { típica }\end{array}$ & K-S & $\mathrm{N}$ & Media & Mediana & $\begin{array}{l}\text { Desv. } \\
\text { típica }\end{array}$ & K-S \\
\hline Amigos & 527 & 3.39 & 4 & 1.302 & $.000^{*}$ & 410 & 3.32 & 3 & 1.286 & $.000 *$ \\
\hline Conciertos en vivo & 528 & 3.15 & 3 & 1.452 & $.000^{*}$ & 404 & 2.95 & 3 & 1.453 & $.000 *$ \\
\hline Familia & 528 & 2.93 & 3 & 1.444 & $.000^{*}$ & 410 & 2.71 & 2.5 & 1.406 & $.000 *$ \\
\hline Iglesia & 523 & 3.25 & 3 & 1.506 & $.000 *$ & 409 & 2.77 & 3 & 1.523 & $.000 *$ \\
\hline Internet & 522 & 3.64 & 4 & 1.453 & $.000 *$ & 404 & 3.72 & 4 & 1.479 & $.000 *$ \\
\hline Radio & 524 & 3.75 & 4 & 1.381 & $.000 *$ & 403 & 3.29 & 4 & 1.552 & $.000 *$ \\
\hline Sitios públicos & 527 & 2.70 & 3 & 1.363 & $.000 *$ & 407 & 2.47 & 2 & 1.311 & $.000 *$ \\
\hline Televisión & 524 & 3.34 & 4 & 1.351 & $.000 *$ & 404 & 3.16 & 3 & 1.424 & $.000 *$ \\
\hline
\end{tabular}

*Estadísticamente significativo al nivel 5\% $(\mathrm{p}<.05)$.

Sobre la variable sexo, radio, Internet, amigos y televisión fueron los agentes más influyentes para las mujeres, mientras que Internet y radio fueron los únicos que obtuvieron mediana 4 para los hombres, siendo los más influyentes en el consumo musical para este sexo. Para verificar las diferencias presentadas entre los sexos que son estadísticamente 
significativas, se optó por emplear la prueba U de Mann-Whitney, adecuada para datos que no obedecen una distribución normal $(\mathrm{p}<.05)$ (ver tabla 2$)$.

Tabla 3. Resultados obtenidos a partir de la prueba U de Mann-Whitney para la variable sexo.

\begin{tabular}{llll}
\hline \multicolumn{1}{c}{ ÍTEM } & \multicolumn{1}{c}{ U de Mann-Whitney } & $\mathrm{Z}$ & $\begin{array}{c}\text { Sig. asintót. } \\
\text { (bilateral) }\end{array}$ \\
\hline Amigos & 103883.000 & -1.037 & .300 \\
Conciertos en vivo & 98177.000 & -2.126 & $.033^{*}$ \\
Familia & 98840.500 & -2.334 & $.020^{*}$ \\
Iglesia & 88154.500 & -4.721 & $.000^{*}$ \\
Internet & 101299.000 & -1.077 & .281 \\
Radio & 88445.500 & -4.402 & $.000 *$ \\
Sitios públicos & 97184.000 & -2.525 & $.012 *$ \\
Televisión & 98746.500 & -1.796 & .072 \\
\hline
\end{tabular}

* Estadísticamente significativo al nivel 5\% $(\mathrm{p}<.05)$.

La prueba U comprobó la existencia de diferencias estadísticamente significativas en función de la variable sexo para los ítems conciertos en vivo, familia, iglesia, radio y sitios públicos, siendo todos más influyentes para los participantes del sexo femenino (ver tabla 3).

Con respecto a la variable edad, se puede destacar la mayor influencia de los medios de comunicación televisión, radio e Internet, conjuntamente a los amigos, en las preferencias de consumo musicales de los adolescentes con edades más tempranas (14 a 18 años) (ver tabla 4).

Tabla 4. Estadísticos descriptivos y test Kolmogorov-Smirnov (K-S) para evaluar la normalidad de la distribución de datos en función de la variable edad.

\begin{tabular}{clllllllll}
\hline Edad & Est. Descrip. & \multicolumn{1}{c}{ TV } & Radio & Familia & Amigos & Iglesia & \multicolumn{1}{c}{ SP } & Conc. en vivo & Internet \\
\hline \multirow{3}{*}{14} & $\mathrm{~N}$ & 78 & 80 & 81 & 80 & 79 & 81 & 81 & 80 \\
\cline { 2 - 10 } & Media & 3.33 & 3.59 & 2.7 & 3.36 & 3.04 & 2.79 & 3.05 & 3.75 \\
\cline { 2 - 10 } & Mediana & 4 & 4 & 2 & 4 & 3 & 3 & 3 & 4 \\
\cline { 2 - 10 } & Desv. Típica & 1.383 & 1.515 & 1.504 & 1.371 & 1.418 & 1.394 & 1.44 & 1.454 \\
\cline { 2 - 10 } & K-S & $.000 *$ & $.000 *$ & $.000 *$ & $.000 *$ & $.000 *$ & $.000 *$ & $.000 *$ & $.000 *$ \\
\hline \multirow{3}{*}{15} & $\mathrm{~N}$ & 202 & 202 & 203 & 203 & 199 & 202 & 200 & 196 \\
\cline { 2 - 10 } & Media & 3.27 & 3.49 & 2.81 & 3.27 & 2.86 & 2.53 & 2.96 & 3.78 \\
\cline { 2 - 9 } & Mediana & 3.5 & 4 & 3 & 3 & 3 & 2 & 3 & 4 \\
\cline { 2 - 9 } & Desv. Típica & 1.428 & 1.474 & 1.37 & 1.258 & 1.508 & 1.258 & 1.428 & 1.463 \\
\cline { 2 - 9 } & K-S & $.000 *$ & $.000 *$ & $.000 *$ & $.000 *$ & $.000 *$ & $.000 *$ & $.000 *$ & $.000 *$ \\
\hline
\end{tabular}




\begin{tabular}{|c|c|c|c|c|c|c|c|c|c|}
\hline Edad & Est. Descrip. & TV & Radio & Familia & Amigos & Iglesia & SP & Conc. en vivo & Internet \\
\hline \multirow{5}{*}{16} & $\mathrm{~N}$ & 302 & 300 & 303 & 303 & 304 & 303 & 302 & 301 \\
\hline & Media & 3.34 & 3.67 & 2.99 & 3.51 & 3.14 & 2.68 & 3.14 & 3.87 \\
\hline & Mediana & 4 & 4 & 3 & 4 & 3 & 3 & 3 & 4 \\
\hline & Desv. Típica & 1.302 & 1.438 & 1.451 & 1.271 & 1.556 & 1.329 & 1.454 & 1.337 \\
\hline & K-S & $.000 *$ & $.000 *$ & $.000 *$ & $.000 *$ & $.000 *$ & $.000 *$ & $.000 *$ & $.000 *$ \\
\hline \multirow{5}{*}{17} & $\mathrm{~N}$ & 251 & 248 & 253 & 253 & 252 & 250 & 251 & 252 \\
\hline & Media & 3.25 & 3.49 & 2.79 & 3.36 & 3.1 & 2.55 & 3.07 & 3.51 \\
\hline & Mediana & 3 & 4 & 3 & 4 & 3 & 2 & 3 & 4 \\
\hline & Desv. Típica & 1.411 & 1.481 & 1.386 & 1.276 & 1.521 & 1.42 & 1.424 & 1.498 \\
\hline & K-S & $.000 *$ & $.000 *$ & $.000 *$ & $.000 *$ & $.000 *$ & $.000 *$ & $.000 *$ & $.000 *$ \\
\hline \multirow{5}{*}{18} & $\mathrm{~N}$ & 63 & 64 & 64 & 64 & 64 & 64 & 64 & 63 \\
\hline & Media & 3 & 3.66 & 2.72 & 3.44 & 3.05 & 2.72 & 3.14 & 3.25 \\
\hline & Mediana & 3 & 4 & 3 & 4 & 3 & 3 & 3.5 & 4 \\
\hline & Desv. Típica & 1.513 & 1.556 & 1.517 & 1.258 & 1.618 & 1.278 & 1.592 & 1.704 \\
\hline & K-S & $.000 *$ & $.000 *$ & $.000 *$ & $.000 *$ & $.000 *$ & $.000 *$ & $.000 *$ & $.000 *$ \\
\hline \multirow{5}{*}{19} & $\mathrm{~N}$ & 19 & 20 & 21 & 21 & 21 & 21 & 21 & 21 \\
\hline & Media & 2.68 & 3.1 & 2.24 & 2.43 & 2.48 & 1.86 & 2.62 & 2.95 \\
\hline & Mediana & 3 & 3 & 2 & 2 & 2 & 2 & 2 & 3 \\
\hline & Desv. Típica & 1.336 & 1.373 & 1.375 & 1.363 & 1.401 & 1.062 & 1.627 & 1.465 \\
\hline & K-S & $.000 *$ & $.000 *$ & $.000 *$ & $.000 *$ & $.000 *$ & $.000 *$ & $.000 *$ & $.000 *$ \\
\hline \multirow{5}{*}{20} & $\mathrm{~N}$ & 13 & 13 & 13 & 13 & 13 & 13 & 13 & 13 \\
\hline & Media & 3 & 29.2 & 2.62 & 2.62 & 3 & 2.31 & 2.92 & 3.62 \\
\hline & Mediana & 3 & 3 & 2 & 2 & 3 & 2 & 3 & 4 \\
\hline & Desv. Típica & 1.472 & 1.656 & 1.805 & 1.66 & 1.871 & 1.601 & 1.706 & 1.66 \\
\hline & K-S & $.000 *$ & $.000 *$ & $.000 *$ & $.000 *$ & $.000 *$ & $.000 *$ & $.000 *$ & $.000 *$ \\
\hline
\end{tabular}

TV $=$ Televisión; $\mathrm{SP}=$ Sitios Públicos. *Estadísticamente significativo al nivel 5\% (p $<.05)$.

A partir del test Kolmogorov-Smirnov, se comprobó que los datos no siguen una distribución normal $(\mathrm{p}<.001)$, siendo necesario la utilización de la prueba Kruskal-Wallis para verificar diferencias existentes entre los grupos de edades (ver tabla 4). Añadido a eso, se llevó a cabo también los test de comparaciones múltiples a partir de la prueba de Dunn-Bonferroni.

Tabla 5. Resultados obtenidos a partir de la prueba Kruskal-Wallis para la variable edad. 


\begin{tabular}{lllll}
\hline \multicolumn{1}{c}{ Ítem } & Chi-cuadrado & Gl & Sig. asintót. & $\begin{array}{c}\text { Comparaciones post-hoc } \\
\text { (Prueba de Dunn-Bonfer- } \\
\text { roni) }\end{array}$ \\
\hline Amigos & 18.448 & 6 & $.005^{*}$ & $\begin{array}{l}14,15,16,17,18>19 \\
16>20\end{array}$ \\
\hline Conciertos en vivo & 3.992 & 6 & .678 & \\
\hline Familia & 9.488 & 6 & .148 & $14,15,16>19$ \\
\hline Iglesia & 7.299 & 6 & .294 & \\
\hline Internet & 17.682 & 6 & $.007^{*}$ & \\
\hline Radio & 8.493 & 6 & .204 & $.048^{*}$ \\
\hline Sitios públicos & 12.692 & 6 & .372 & \\
\hline Televisión & 6.473 & 6 & $.05) .17$ & \\
\hline
\end{tabular}

*Estadísticamente significativo al nivel $5 \%(\mathrm{p}<.05)$.

En la tabla 5, se observa que los resultados apuntaron a diferencias estadísticamente significativas para 2 de los 8 ítems evaluados: amigos e Internet. La influencia de los amigos se mostró más importante para alumnos de edades más tempranas, teniendo un impacto más grande para el grupo de 16 años. El Internet también se mostró más relevante para los grupos de edades más jóvenes, con destaque para aquellos entre 14 y 16 años.

\section{Discusiones y conclusiones}

La fuerte influencia del Internet y de la radio en los adolescentes ya había sido señalada en estudios anteriores (Cremades, 2008; Ter Bogt, Engels, Bogers y Kloosterman, 2010) y confirma la presencia sobresaliente de los medios de comunicación de masas en la vida de estos estudiantes. Sin embargo, el elevado índice de respuestas para el ítem amigos dialoga con los resultados indicados por Ho (2002) y Finnas (1989b), afirmando este último autor que los adolescentes suelen escuchar música solos o con sus amigos. Este resultado lleva a pensar que aspectos del conglomerado social tienden a suavizar el impacto de los mass media, no tomándoles como únicas referencias para el consumo musical (Carvalho, 1999).

Sobre la variable sexo, la prueba U comprobó la existencia de diferencias estadísticamente significativas para los ítems conciertos en vivo, familia, iglesia, radio y sitios públicos, siendo todos más influyentes para los participantes del sexo femenino. Eso demuestra la mayor predisposición de las mujeres para la música (Crowther y Durkin, 1982; North y Hargreaves, 2008; White, 2001), recibiendo influencias de casi todos los agentes de 
educación informal evaluados, resultados que en parte poseen convergencia con el estudio de Cremades (2008).

Con respecto a la variable edad, la prueba Kruskal-Wallis arrojó diferencias estadísticamente significativas para los ítems amigos e Internet. La influencia de los amigos se mostró más importante para alumnos de edades más tempranas, teniendo un impacto más grande para el grupo de 16 años, lo que puede tener relación con la necesidad de fortalecimiento de las relaciones afectivas entre los adolescentes (Pimentel, 2004). Pais (1998) afirma que la música consumida (así como el vestuario, la apariencia y el lenguaje) sí se constituye como 'elemento simbólico' que da coherencia interna a los grupos de iguales, sirviendo para formar y consolidar tanto una identidad grupal como para diferenciarlos de otros grupos.

La Internet también se mostró más relevante para los grupos de edades más jóvenes, con destaque para aquellos entre 14 y 16 años. Este resultado puede estar relacionado, primeramente, con el proceso de inclusión digital escolar por lo cual las escuelas brasileñas han pasado recientemente y con la mayor facilidad de los alumnos con edades más tempranas en el manejo de herramientas tecnológicas (Costa, 2004; Pereira y Silva, 2009). Por otro lado, es importante destacar que la Internet se ha convertido en la media característica de los adolescentes, siendo uno de sus principales medios de contacto con el mundo exterior, creando muchas veces una realidad particular a partir de las redes sociales, utilizando esta herramienta también para el desarrollo de habilidades múltiples, como tocar un instrumento musical, hablar un segundo idioma, conocer particularidades de lugares exóticos, etc. Por lo tanto, se puede afirmar que la opción por la Internet se asemeja con el propósito de libertad de los adolescentes, dato comprobado a partir del test de correlación de Spearman donde esta herramienta presentó correlación positiva y moderada para el ítem conciertos en vivo $(r=.463 ; p<.001)$, demostrando la necesidad del adolescente de elegir su propio tipo de música. Internet y los conciertos en vivo dependen de la voluntad intrínseca del individuo para la toma de decisión, optando por cuáles conciertos asistir, qué tipo de página acceder, entre otras.

Por último, fue posible comprobar la hipótesis sobre la importancia de los mass media en el consumo musical de los adolescentes, constituyéndose como el principal agente de influencia de la educación informal en la construcción de las preferencias musicales de dichos adolescentes. El poder de los mass media no está limitado al consumo musical, sino que abarca también el tipo de lenguaje, vestuario, comportamiento, sexualidad, opinión y personalidad de los adolescentes, en especial aquellos que viven en sitios de menor poder económico (Quadros Jr. y Lorenzo, 2013), lo que despierta la preocupación 
e interés para discutir en otro momento sobre la formación del ciudadano en el siglo XXI. El resultado obtenido en este estudio pone de manifiesto la urgente necesidad de buscar medidas de democratización del acceso a la información que permitan al ciudadano común una programación musical y cultural más diversificada, y que posibilite su formación de manera plural, tomando consciencia sobre sus decisiones, lo que desea consumir, y su cotidianidad.

\section{Referencias}

Adorno, T., \& Horkheimer, M. (1985). Dialética do esclarecimento: fragmentos filosóficos. Rio de Janeiro: Jorge Zahar Ed.

Barbero, M., Vila, E., \& Suárez, J. (2003). Psicometría. Madrid: Universidad Nacional de Educación a Distancia.

Calabre, L. (2002). A era do rádio. Rio de Janeiro: Jorge Zahar Ed.

Canclini, N. (2000). Culturas híbridas: estratégias para entrar e sair da modernidade. São Paulo: Edusp.

Cárdenas Soler, R., Martínez Álvarez, J., \& Cremades Andreu, R. (2017). Competencias de lectura y escritura en música. Una propuesta para su asimilación en el currículo escolar. Cuadernos de Lingüística Hispánica, (29), 181-201. doi: https://doi.org/10.19053/0121053X. n29.2017.5859

Carvalho, J. (1999). Transformações da sensibilidade musical contemporânea. Horizontes Antropológicos, 11, 53-91.

Costa, F. (2004). 0 que justifica o fraco uso dos computadores na escola? Polifonía, 7, 19-32.

Costa, A. et al. (2012). Pedagogia, motivação e desenvolvimento empresarial. Factu Ciência, 22,143-165.

Cremades, R. (2008). Conocimiento y preferencia sobre los estilos musicales en los estudiantes de Educación Secundaria Obligatoria en la Ciudad Autónoma de Melilla (Tesis Doctoral). Universidad de Granada, Granada, España. Recuperado de: http://digibug.ugr.es/ bitstream/10481/2023/1/17632468.pdf

Crowther, R. y Durkin, K. (1982). Sex- and Age-Related Differences in the Musical Behaviour, Interests and Attitudes Towards Music of 232 Secondary School Students. Educational Studies, 8(2), 131-139.

Díaz, F. (2010). Uso educativo de la televisión para la prevención de la violencia infantil. En J. Domingo (Coord.), El niño y la televisión (pp. 177- 180). Granada: Universidad de Granada. 
Fernández, F. (2010). Las dictaduras de la sociedad de la información. En J. Domingo (Coord.), El niño y la televisión (pp. 95-102). Granada: Universidad de Granada.

Finnäs, L. (1989a). A comparison between young people's privately and publicly expressed musical preferences. Psychology of music, 17, 132-145. doi: 10.1177/0305735689172004

Finnäs, L. (1989b). How can musical preferences be modified? Bulletin of the Council for Research in Music Education, 102, 1-58.

Fittipaldi, V. (2005). Musicalização através do teclado e as novas tecnologias do século XXI (Tesis de Maestría). Universidade Federal do Estado do Rio de Janeiro, Rio de Janeiro, Brasil. Recuperado de: http://www.dominiopublico.gov.br/pesquisa/DetalheObraForm.do?select_ action $=\&$ co_obra $=40474$

Foucault, M. (1979). Microfísica do poder (R. Machado, Trad.). Rio de Janeiro: Edições Graal.

Foucault, M. (2008). Vigiar e punir: nascimento da prisão (R. Ramalhete, Trad.) (35a. ed.). Petrópolis: Vozes.

García, Á., \& López, A. (2010). La influencia de la televisión en los niños. Opiniones de los futuros enseñantes. En J. Domingo (Coord.), El niño y la televisión (pp. 207-213). Granada: Universidad de Granada.

Gunter, B. (2005). Trust in the news on television. Aslib Proceedings: New Information Perspectives, 57(5), 384-397.

Hargreaves, D., \& North, A. (1997). The Social Psychology of Music. Oxford: Oxford University Press.

Henriques, M. (2005). Comunicação, comunidades e os desafios da mobilização social. Actas del Congresso Brasileiro de Ciências da Comunicação, 28, 1-14. Rio de Janeiro: UERJ.

Ho, W. (2002). Musical behavior of young Hong Kong students. Educational Research Journal, 17(2), 199-217.

Laraia, R. (2002). Cultura: um conceito antropológico. Rio de Janeiro: Jorge Zahar Editor.

Legorburu, J. (2001). Utilidady eficacia de la comunicación radiofónica en el proceso educativo (Tesis Doctoral). Universidad Complutense de Madrid, Madrid, España. Recuperado de: http://eprints.ucm.es/4626/

Leung, C. (2004). Curriculum and culture. A model for content selection and teaching approaches in music. British Journal Music Education, 21, 25-39. doi: 10.1017/\$0265051703005503

Levine, J., \& Russo, E. (1987). Majority and Minority Influence. En C. Hendrick (Ed.), Review of personality and social psychology (pp. 13-54). Thousand Oaks, CA, US: Sage Publication. 
Levitin, D. (2007). Life soundstracks: the uses of music in everyday life. Recuperado de: http:// www.russballard.com/rbv7-workshop/physics/Unit\%203\%20Vibrations/LifeSoundtracks. pdf.

Liarte, J. (2000). Viaje musical por Internet. Eufonía, 19, 67-85.

Lorenzo, 0. (2002). Educación musical no formal a través de la prensa de divulgación general en España (Tesis de Doctorado). Universidad Nacional de Educación a Distancia, Madrid, España. Recuperado de: http://tesis.com.es/documentos/educacion-muscial-no-formaltraves-prensa-divulgacion-general-espana/

Lorenzo, 0. (2003). Reflexiones en torno a la cultura y la educación. En L. Herrera, O. Lorenzo, M. Mesa, y I. Alemany (Coords.). Intervención psicoeducativa: Una perspectiva multidisciplinar (pp. 10-22). Granada: Grupo Editorial Universitario.

Lorenzo, 0. (2004). Fundamentos y retos de educación social-informal desde el análisis crítico de los medios de comunicación. Biblioteca Digital de la Organización de Estados Iberoamericanos para la Educación, la Ciencia y la Cultura. Tecnología Educativa. Recuperado de: http://www.oei.es/oeivirt/tecnologiaeducativa.htm

Lorenzo, 0., \& Cremades, R. (2008). Educación formal y conocimiento sobre estilos musicales en estudiantes de educación secundaria obligatoria. Eufonía, 42, 81-88.

Lorenzo, 0., Herrera, L., \& Cremades, R. (2008). Investigación sobre preferencias de Estilos Musicales en estudiantes españoles de Educación Secundaria Obligatoria. En M. Ortiz (Coord.), Música. Arte. Diálogo. Civilización (pp. 301-332). Coimbra (Portugal): Center for Intercultural Music Arts y Grupo de Investigación HUM-742.

McLuhan, M. (1996). Comprender los medios de comunicación: las extensiones del ser humano. Barcelona: Paidós Comunicación.

Merrill, J., Lee, J., \& Friedlander, E. (1991). Medios de comunicación social. Teoría y práctica en Estados Unidos y en el mundo. Madrid: Fundación Germán Sánchez Ruipérez.

Monteiro, L. (2001). A internet como meio de comunicação: possibilidades e limitações. Actas del Congresso Brasileiro de Comunicação, 24, 27-37.

North, A., \& Hargreaves, D. (1997). Experimental aesthetics and everyday music listening. En D. Hargreaves y A. North, The Social Psychology of Music (pp. 84-103). Oxford: Oxford University Press.

North, A., \& Hargreaves, D. (1999). Music and adolescent identity. Music Education Research, 1, 75-92. doi: 10.1080/1461380990010107 
North, A., \& Hargreaves, D. (2008). The Social \& Applied Psychology of Music. New York: Oxford University Press.

Pais, J. (1998). Culturas juvenis. Lisboa: Imprensa Nacional Casa da Moeda.

Pereira, M., \& Silva, B. (2009). A relação dos jovens com as TIC e o factor divisão digital na aprendizagem. Actas del X Congresso Internacional Galego-Português de Psicopedagogia, 10, 5408-5431. Braga: Universidade do Minho.

Pimentel, C. (2004). Valoreshumanos, preferencia musical, identificação grupale comportamento anti-social (Tesis de Maestría). Universidade Federal da Paraíba, Paraíba, Brasil.

Quadros Jr., J. (2013). Preferencias musicales en estudiantes de enseñanza secundaria en Brasil: El caso de la ciudad de Vitória, Espírito Santo (Tesis Doctoral). Universidad de Granada, Granada, España.

Quadros Jr., J., \& Lorenzo, 0. (2010). Preferências musicais em estudantes de ensino médio no Brasil: o caso de Vitória, Espírito Santo. Música Hodie, 10(1), 109-128.

Quadros Jr., J., \& Lorenzo, 0. (2013). Preferência musical e classe social: um estudo com estudantes de ensino médio de Vitória, Espírito Santo. Revista da ABEM, 21(31), 35-50.

Quadros Jr., J., \& Lorenzo, 0. (2015). Educación informal y hábitos de consumo musical en enseñanza media en Brasil. En L. Herrera Torres y M. Ortiz Molina (Coords.), Desarrollo, educación, diversidade y cultura: análisis interdisciplinar (pp. 123-137). Coimbra: Fernando Ramos Editor.

Quadros Jr., J., \& Lorenzo, 0. (2016). Preferência Musical: uma revisão dos fatores extramusicais que influenciam na escolha de músicas por ouvintes. En P. Molinari (Org.), Música, educação e cultura: tessituras e tecituras no nordeste brasileiro (en prensa). São Paulo: Editora Faccamp. Recuperado de: http://www.faccamp.br

Radocy, R. (1975). A naive minority of one and deliberate majority mismatches of tonal stimuli. Journal of Research in Music Education, 23, 120-133. doi: 10.1177/030573567531003

Santos, L. (2006). Educação Musical nos anos iniciais do ensino fundamental: concepções $e$ ações coordenadoras pedagógicas escolares (Tesis de Maestría). Universidade Federal de Santa Maria, Santa Maria, Brasil. Recuperado de: http://www.dominiopublico.gov.br/ pesquisa/DetalheObraForm.do?select_action $=\&$ co_obra $=40462$

Saperas, E. (1987). Los efectos cognitivos de la comunicación de masas. Barcelona: Ariel.

Sarramona, J. (1994). Fundamentos de Educación. Barcelona: CEAC. 
Schäfer, T. (2008). Determinants of music preference (Tesis de Maestria). Technischen Universität Chemnitz, Chemnitz, Deutschland. Recuperado de: http://www.qucosa.de/fileadmin/data/ qucosa/documents/5749/data/DissertationThomasSchaefer.pdf

Silva, J. (2006). A influência dos meios de comunicação social na problemática da escolha profissional: o que isso suscita à Psicologia no campo da orientação vocacional/profissional? Psicologia: ciência e profissão, 24(4), 60-67.

Silva, R. (2012). 0 potencial da comunicação como instrumento de estímulo à educação ambiental no interior do Estado de São Paulo. Revista ALTERJOR, 2(6), 1-15.

Slater, M., \& Henry, K. (2013). Prospective Influence of Music-Related Media Exposure on Adolescent Substance-Use Initiation: A Peer Group Mediation Model. Journal of Health Communication: International Perspectives, 18(3), 291-305. doi: 10.1080/10810730.2012.727959

Telebrasil (2016). Relatórios séries temporais customizados. Recuperado de http://www.telebrasil. org.br/estatisticas/panorama-do-setor

Teleco (2012). Estatísticas do Brasil. Recuperado de: http://www.teleco.com.br/estatis.asp

Telecom (2016). International Telecommunication Union. Recuperado de: http://www.itu.int/ ITU-D/ict/statistics/

Ter Bogt, T., Engels, R., Bogers, S., \& Kloosterman, M. (2010). "Shake it baby, shake it": media preferences, sexual attitudes and gender stereotypes among adolecents. Sex Role, 63, 844859. doi 10.1007/s11199-010-9815-1

Vázquez, M. (1997). Historia y Comunicación Social. Barcelona: Grijalbo Mondadori.

White, C. (2001). The effects of class, age, gender and race on musical preferences: an examination of the omnivore/univore framework (Tesis de Maestría). Faculty of the Virginia Polytechnic and State University, Virginia, EE.UU. Recuperado de http://scholar.lib. vt.edu/theses/available/etd-09072001-101445/unrestricted/whitethesis.doc.pdf 